\title{
Reconocimiento geológico de la Formación La Luna en el sector de Matanza (Oeste del Macizo de Santander, Colombia)
}

\author{
German David Patarroyo-Camargo ${ }^{1,2^{*}} \mathbb{D}$; Carlos Mario Alarcón-Gómez ${ }^{1} \mathbb{D}$; José Manuel Torres-Parada ${ }^{1}$; \\ Jefferson Steven Díaz-Villamizar ${ }^{1}$; ; Juan Sebastián Gómez-Coronado ${ }^{1}$ iD; José Javier Márquez-Prada ${ }^{1}$ iD; \\ Liz Andrea Pontón-Parada ${ }^{3}$ iD; Diana Marcela Barragán-Martinez ${ }^{3}$
}

Forma de citar: Patarroyo-Camargo, G.D.; Alarcón-Gómez, C.M.; Torres-Parada, J.M.; Díaz-Villamizar, J.S.; Gómez-Coronado, J.S.; Márquez-Prada, J.J.; Pontón-Parada, L.A.; Barragán-Martinez, D.M. (2021). Reconocimiento geológico de la Formación La Luna en el sector de Matanza (Oeste del Macizo de Santander, Colombia). Boletín de Geología, 43(1), 35-51. https://doi.org/10.18273/revbol.v43n1-2021002

\begin{abstract}
Resumen
Este trabajo abarcó el estudio sedimentológico, paleontológico y geoquímico de la Formación La Luna, en una localidad al oeste del Macizo de Santander (Matanza, Colombia).

La sección corresponde a un corte de 42 metros, compuesto por una alternancia de mudstones y wackestones interestratificados, niveles de chert, fosforitas y bentonitas. El contenido fósil está conformado por restos de amonitas, inocerámidos, gasterópodos turritélidos, fragmentos de peces y foraminíferos planctónicos. La asociación fósil indica una edad de Coniaciano-Santoniano. Las características sedimentológicas, el tipo de fósiles encontrados y los análisis petrográficos/geoquímicos desarrollados en esta sección sugieren condiciones de depositación en plataforma externa y una baja oxigenación en los sedimentos de fondo.

Las conclusiones de este trabajo coinciden con interpretaciones preexistentes de la región cuenca, sugiriendo relaciones litoestratigráficas con la sucesión sedimentaria del Valle Medio del Magdalena (parte superior del "Miembro Galembo"). Sin embargo, solo estudios estratigráficos detallados en esta parte del Macizo de Santander, permitirán entender su evolución geológica durante el Cretácico superior.
\end{abstract}

Palabras clave: Cretácico superior; Litoestratigrafía; Formación La Luna; Macizo de Santander; Paleontología.

\section{Geological evaluation of La Luna Formation at the Matanza area (west Santander Massif, Colombia)}

\begin{abstract}
This study comprised the sedimentological, paleontological and geochemical survey of the La Luna Formation, using as a reference one section in the west flank of the Santander Massif (Matanza, Colombia).

The 42-meter length section comprises an alternance of interstratified mudstones, wackestones, chert, phosphorite and bentonite levels. Fossil content is defined by rest of ammonoids, inoceramids, turritellid gastropods, fish remains and planktonic foraminifers, suggesting all a Coniacian-Santonian age. The facial analysis, the fossil content and preliminary petrographic/geochemical analysis also suggest an outer shelf setting and low oxygenation conditions in the bottom sediments.

In general, our results agree with previous interpretations of La Luna Formation, suggesting a closer relation with the Upper Cretaceous succession of the Middle Magdalena (upper "Galembo Member"). However, only detailed stratigraphical studies in this part of the Santander Massif, will allow a better understanding of its geological evolution during the Late Cretaceous.
\end{abstract}

Keywords: Upper Cretaceous; Lithostratigraphy; La Luna Formation; Santander Massif; Paleontology.

${ }^{1}$ Semillero de Paleontología y Bioestratigrafía, Universidad Industrial de Santander, Bucaramanga, Colombia. (*) paleo.patarroyo@gmail.com; cmag_1996@hotmail.com; manutorresgeo@gmail.com; yeyediaz16@hotmail.com; sebastian_2496@hotmail.com; josmark1997@hotmail.com

${ }^{2}$ Stratos Consultoría Geológica, Bucaramanga, Colombia.

${ }^{3}$ Fundación Universitaria del Área Andina, Valledupar, Colombia. lponton@estudiantes.areandina.edu.co; dbarragan6@estudiantes.areandina.edu.co 


\section{Introducción}

Por su potencial petrolífero, la Formación La Luna corresponde a una de las unidades estratigráficas más estudiadas dentro de las sucesiones cretácicas del norte de Suramérica (Talukdar y Marcano, 1994; Mann et al., 2006). Definida por Garner (1926) en una quebrada homónima en el Perijá venezolano, la Formación La Luna está conformada por una sucesión de calizas y shales bituminosos con concreciones, al igual que niveles fosfáticos y glauconíticos hacia su parte superior. Su espesor para la región de la cuenca de Maracaibo oscila entre los 100-300 m y debido a su heterogeneidad litológica, distintas subdivisiones estratigráficas han sido propuestas (miembros Chejendé, Timbetes, Tres Esquinas, entre otros). El rango de edad propuesto comprende el intervalo Cenomaniano tardío-Campaniano, definido a partir de quimioestratigrafía, así como con el registro de amonitas, bivalvos y microfósiles (foraminíferos, nanofósiles; Renz, 1982; Martinez y Hernandez, 1992; Erlich et al., 1999; De Romero et al., 2003; Machado et al., 2016). En términos generales, la depositación de las sedimentitas de la Formación La Luna, y unidades estratigráficas equivalentes por fuera de Venezuela (Formación La Frontera, Formación La Luna sensu Morales et al., 1958, Grupo Napo, Formación Chonta) se dio bajo condiciones anóxicas-disóxicas en un mar epicontinental (profundidades máximas entre 300-500 m) de amplia distribución geográfica ("La Luna Sea"; Villamil y Arango, 1998; Villamil y Pindell, 1998; Erlich et al., 2000; Bayona, 2018; Sarmiento, 2018).

Esta amplia distribución lleva a que la denominación de Formación La Luna haya sido utilizada para sucesiones del Cretácico superior en La Guajira (Renz, 1960), la cuenca del Catatumbo (Pedraza y Ramírez, 2011; Patarroyo et al., 2017), o las reportadas en el Valle Medio del Magdalena (sensu Morales et al., 1958; Rangel et al., 2000; Sarmiento, 2011; Sarmiento et al., 2015; Bayona, 2018). Aunque las interpretaciones cronoestratigráficas y paleoambientales observadas en Colombia concuerdan en gran medida con las descritas para Venezuela, el uso del término Formación La Luna en zonas como el Valle Medio del Magdalena no ha estado exento de discusión. Por ejemplo, Terraza-Melo (2019) aboga por el abandono de la Formación La Luna sensu stricto, además de elevar a unidades formacionales los miembros Salada, Pujamana y Galembo, siguiendo la propuesta original de Wheeler (1929). De igual forma, propone una nueva unidad litoestratigráfica para el segmento más superior, compuesto principalmente de niveles fosfáticos (Formación La Renta).
La definición de la Formación La Luna en otros sectores de la Cordillera Oriental, como las áreas proximales al Macizo de Santander, presenta inconvenientes adicionales. Esto, debido a que, si bien, estudios regionales dan prueba de su presencia (Vargas et al., 1981), no es claro si sus características litológicas y contenido fósil pueden ser asociadas con la litoestratigrafía de la región del Catatumbo, donde se podría hablar de la Formación La Luna per se, o existen similitudes con la litoestratigrafía del Valle Medio del Magdalena, y su propuesta de nomenclatura estratigráfica más reciente (Terraza-Melo, 2019).

Este trabajo presenta los resultados del análisis litológico, geoquímico y paleontológico de una sección del Cretáceo superior, ubicada en el sector de Matanza (oeste del Macizo de Santander) y cartografiada como Formación La Luna (Ward et al., 1973; 1977). A través de dicho análisis se busca: 1) establecer si las sucesiones del Cretácico superior del flanco oeste del Macizo de Santander presentan similitudes con las unidades estratigráficas del Valle Medio del Magdalena o con las de la Cuenca del Catatumbo; 2) ampliar la línea base de conocimiento de la cobertera sedimentaria cretácica que colinda con el Macizo de Santander.

\section{Marco geológico}

El Macizo de Santander se encuentra ubicado en la parte norte de la Cordillera Oriental Colombiana. $\mathrm{Su}$ basamento se compone principalmente de rocas metamórficas de edad Neoproterozoica (Neis de Bucaramanga) y Ordovícico temprano (Esquistos del Silgará y Ortoneis), que representan eventos de metamorfismo relacionados con las orogenias Grenvilliana y Famatiniana (Cordani et al., 2005; Restrepo-Pace y Cediel, 2010; Van der Lelij, 2013; Mantilla-Figueroa et al., 2013, 2016). El basamento metamórfico es intruido por granitoides de finales del Triásico e inicios del Jurásico temprano (Goldsmith et al., 1971; Ward et al., 1973; Dörr et al., 1995; Van der Lelij, 2013; Mantilla-Figueroa et al., 2013).

A finales del Triásico y durante el Jurásico, la región que rodea al macizo se caracterizó por una tectónica extensiva y formación de estructuras tipos graben (Mojica y Kammer, 1995; Mojica et al., 1996; Bayona et al., 2020) las cuales estuvieron acompañadas con una sedimentación continental sintectónica (Kammer y Sánchez, 2006; Alarcón y Rodríguez, 2019) evidenciada en el registro estratigráfico de las formaciones Bocas, Jordán y Girón. 
La cobertera sedimentaria cretácica asociada al Macizo de Santander, descansa en inconformidad estratigráfica o en contactos fallados con el basamento ígneo-metamórfico. Esta sucesión inicia con el registro continental fluvial de la Formación Los Santos (Berriasiano), previo a la transgresión marina que da origen a la sedimentación del intervalo formacional Rosablanca-Tablazo, en un lapso que comprende desde el Valanginiano hasta finales del Albiano (Ward et al., 1973; Etayo-Serna y Rodríguez, 1985; Cooper et al., 1995).

La depositación de las sedimentitas que definen a la Formación La Luna comienza desde finales del Cenomaniano y continúa en el TuronianoSantoniano, cuando hay un evento transgresivo de gran envergadura que establece un dominio oceánico en el norte de Suramérica. La mayoría de los fondos marinos correspondieron a facies finas con carbonatos y abundante materia orgánica, posiblemente formados en una plataforma externa con condiciones anóxicas (Santana, 1986; Martinez y Hernandez, 1992; Erlich et al., 1999; Villamil et al., 1999; Bayona, 2018; Sarmiento, 2018). Adicionalmente, se presentó la depositación de niveles de bentonitas, específicamente para el intervalo Turoniano-Santoniano. Estas capas se pueden seguir de manera regional en el VMM y se han reportado sobre el margen SW del Macizo de Santander (Villamil y Arango, 1998; Vásquez et al., 2010; Terraza-Melo, 2012; Ballesteros et al., 2013; Sarmiento et al., 2015). Finalmente, a partir del Campaniano la aparición de altos intraplaca al oriente de la Cordillera Central, y el retroceso del nivel del mar llevaron a la progradación de la sucesión sedimentaria hacia finales del Maastrichtiano y el Paleoceno (formaciones Umir, Lisama; Sarmiento et al., 2015; Sarmiento, 2018; Bayona, 2018; Bayona et al., 2020).

Para el caso puntual de las sucesiones sedimentarias del flanco oeste del Macizo de Santander, estas se encuentran fuertemente deformadas por la incidencia del sistema de fallas del Río Suratá, el cual las pone en contacto fallado con el basamento cristalino (Figura 1).
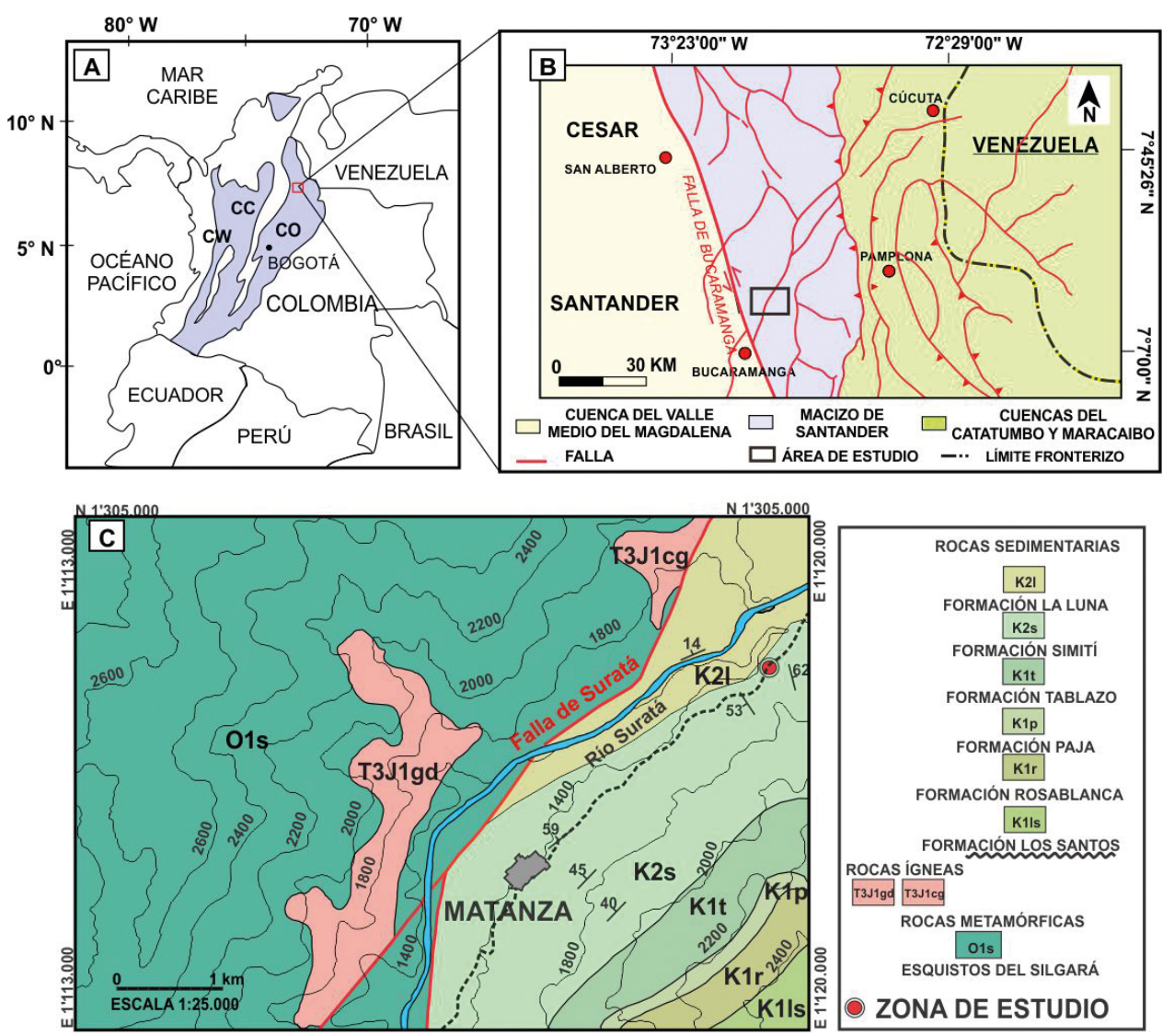

Figura 1. A-B. Localización de la sección estudiada. CO-Cordillera Oriental, CC-Cordillera Central, CW-Cordillera Occidental. Zona de estudio en el recuadro. C. Mapa geológico del área de estudio (modificado de Ward et al., 1977). 
La Formación La Luna, cuyo espesor Ward et al. (1973) estiman menor a los observados en el Valle Medio del Magdalena ( 300 m), aflora en distintos bloques fallados al norte de Charta y el oeste de Matanza, al igual que sobre la vía que conduce de Suratá a California. El afloramiento estudiado corresponde a un corte de 42 metros sobre la vía que conduce de Matanza a Suratá, en el sector de "La Cantera" (7²0'19,50" N, 7259'41,42” W; Figura 1C). Dado el espesor levantado, se estima que la sección estudiada corresponde a solo un segmento de la unidad, probablemente de la parte media-superior de la Formación La Luna.

\section{Metodología}

En la sección estudiada se elaboró una columna estratigráfica a escala 1:10, siguiendo la metodología descrita en el Proyecto Cretácico (Etayo-Serna, 1985). Las rocas carbonatadas fueron clasificadas en campo bajo la propuesta de Dunham (1962) y el espesor de estratos se describió de acuerdo con los definidos por Campbell (1967). Igualmente, para la clasificación del color se utilizó la carta de colores de rocas (Goddard, 2009). Para el reconocimiento preliminar de los invertebrados fósiles se tomaron como guía distintos trabajos del Cretácico superior en Colombia (Villamil y Arango, 1998; Patarroyo, 2011).

Con el objeto de tener una valoración sedimentológica y bioestratigráfica de la sección, se recolectaron muestras para análisis petrográficos $\mathrm{y}$ micropaleontológicos (foraminíferos, palinología). Las muestras de petrografía (4 muestras) fueron preparadas en el Laboratorio de Procesamiento de Secciones Delgadas de la Fundación Universitaria del Área Andina, y posteriormente se analizaron en el Laboratorio de Petrografía de la Universidad Industrial de Santander (UIS). Se llevó a cabo una descripción de las características texturales y composicionales de cada muestra, mientras que la descripción de la porosidad en las rocas carbonatadas se elaboró teniendo en cuenta lo planteado por Choquette y Pray (1970). Posteriormente, se llevó a cabo un conteo de 200 a 300 puntos para finalmente clasificar las rocas según la nomenclatura de Folk (1962).

Las muestras de micropaleontología y palinología (5 muestras), fueron procesadas en Paleoflora Limitada y Stratos Consultoría Geológica, siguiendo protocolos estándar para material del Cretácico (Traverse, 2007; Sohn, 1961). Para la identificación taxonómica de las especies de foraminíferos planctónicos se consultó lo propuesto por Caron (1985), Nederbragt (1991), Georgescu (2005) y Premoli-Silva y Verga (2011).

En la parte correspondiente a la geoquímica se analizaron 10 muestras para estimar el contenido de Carbono Orgánico Total (COT), usando un analizador de carbono y azufre LECO SC-144DR (Tabla 1). Los análisis fueron realizados en el Laboratorio de Geoquímica del Petróleo de la UIS (Sede Guatiguará).

Tabla 1. Relación de muestras colectadas para cada análisis.

\begin{tabular}{|c|c|c|c|c|c|}
\hline Muestra & $\begin{array}{c}\text { Altura } \\
\text { Estratigráfica } \\
\text { (m) }\end{array}$ & Litología & Petrografía & Micropaleontología & Micropaleontología \\
\hline MAT-1 & 41,8 & Mudstone & $\mathrm{x}$ & $\mathrm{x}$ & $\mathrm{x}$ \\
\hline MAT-2 & 40 & Mudstone & & & \\
\hline MAT-3 & 38,3 & Mudstone & $\mathrm{x}$ & $\mathrm{x}$ & $\mathrm{x}$ \\
\hline MAT-4 & 37,4 & Mudstone & & & \\
\hline MAT-6 & 34,4 & Lodolita calcárea & & & \\
\hline MAT-7 & 34,1 & Lodolita calcárea & $\mathrm{x}$ & $\mathrm{x}$ & $\mathrm{x}$ \\
\hline MAT-10 & 29 & Lodolita calcárea & & & \\
\hline MAT-13 & 24,3 & Mudstone & & & \\
\hline MAT-14 & 23,8 & Chert & & & \\
\hline MAT-16 & 20 & Mudstone & $\mathrm{x}$ & $\mathrm{x}$ & $\mathrm{x}$ \\
\hline MAT-17 & 19 & Wackestone & $\mathrm{x}$ & $\mathrm{x}$ & $\mathrm{x}$ \\
\hline MAT-19 & 7,5 & Chert & & & \\
\hline
\end{tabular}




\section{Resultados}

En el área de estudio, el tramo aflorante de la Formación La Luna puede ser subdividido en cuatro segmentos, que en total tienen un espesor de $42 \mathrm{~m}$ (Figura 2).

\section{Descripción litológica}

Segmento I $(8,66 \mathrm{~m})$ : la base del segmento está constituida por una capa de wackestone de geometría tabular de $30 \mathrm{~cm}$ de espesor, en contacto neto con capas delgadas a medianas de mudstones color gris claro (N7) a gris oscuro (N3), inter-estratificadas con niveles delgados de chert negro (N1). El contacto entre este tipo de niveles es onduloso en la parte inferior y neto en la parte superior del segmento. Algunas capas de mudstones presentan geometría lenticular y otras tabulares, desarrollando laminación planoparalela. Asimismo, las capas de mudstones presentan restos de peces, amonitas, bivalvos y gasterópodos. En la parte media del segmento se tienen capas delgadas $(\sim 3 \mathrm{~cm})$ de bentonitas color marrón amarillento (10YR 5/4) en contacto neto con capas de chert y capas medias de mudstones con concreciones de hasta $45 \mathrm{~cm}$ de diámetro. En estas capas se identifican restos esqueletales de escamas de peces y fragmentos carbonosos. Los restos de moluscos están presentes a lo largo del segmento, tanto en las concreciones como en los mudstones. Hacia el techo del segmento afloran capas delgadas de lodolitas físiles y los niveles de chert presentan un espesor variable, tendiendo a acuñarse (Figura 3A).

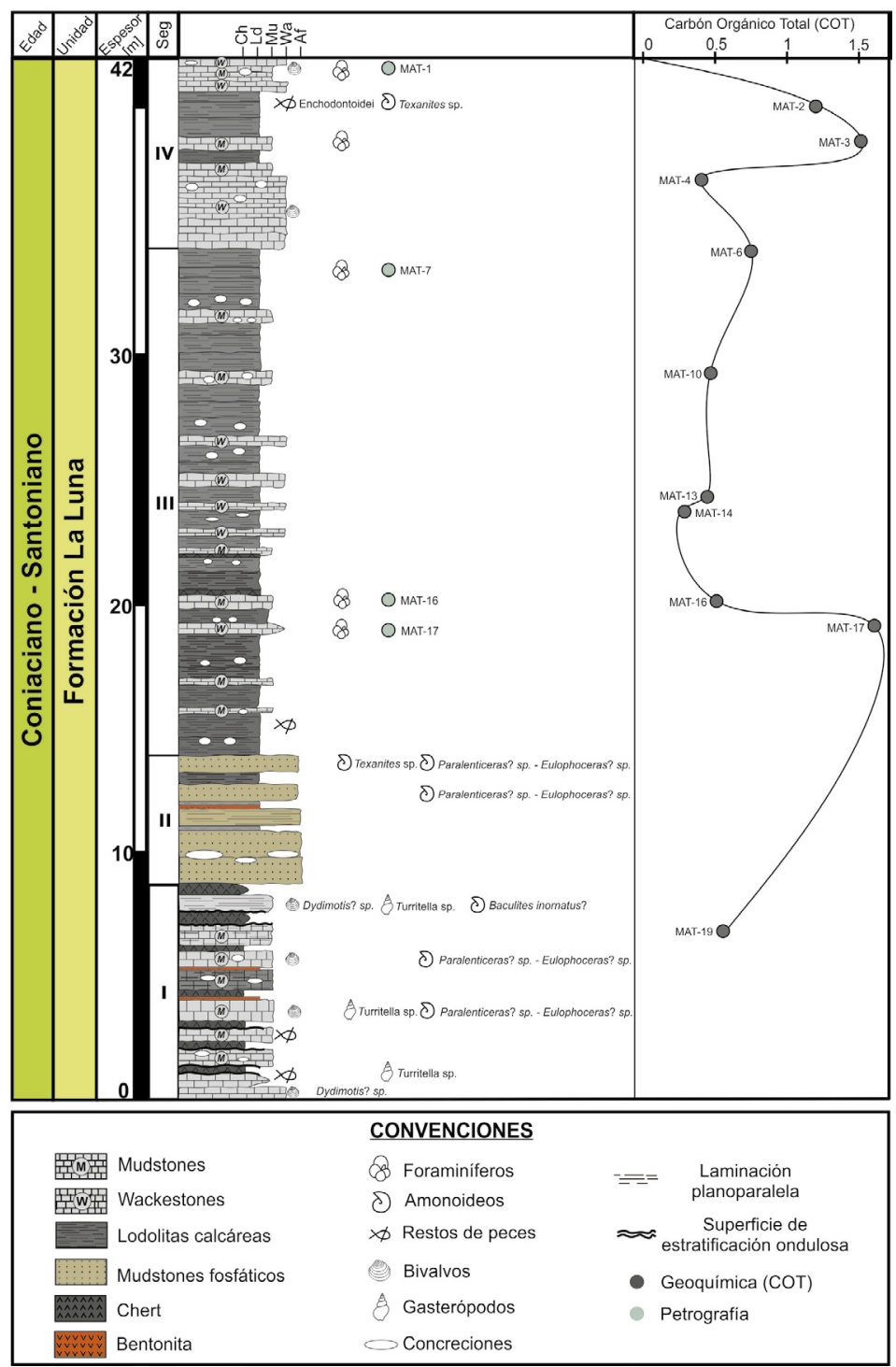

Figura 2. Columna estratigráfica de la Formación La Luna en el sector de Matanza. 
Segmento II $(5,17 \mathrm{~m})$ : este segmento está constituido por capas delgadas a medias de mudstones fosfáticos color gris medio (N4-N5) y lodolitas calcáreas silicificadas de color gris verdoso claro (5G 8/1) en el techo. La geometría de las capas es mayormente tabular. En la parte inferior del segmento se encuentran concreciones calcáreas entre 70 y $90 \mathrm{~cm}$ de diámetro, con presencia de restos de amonitas, mientras que hacia la parte media del segmento algunos mudstones fosfáticos desarrollan laminación planoparalela (Figura 3B). Al igual que en el segmento I, se identificó una capa de bentonita de $3 \mathrm{~cm}$ hacia la parte media.
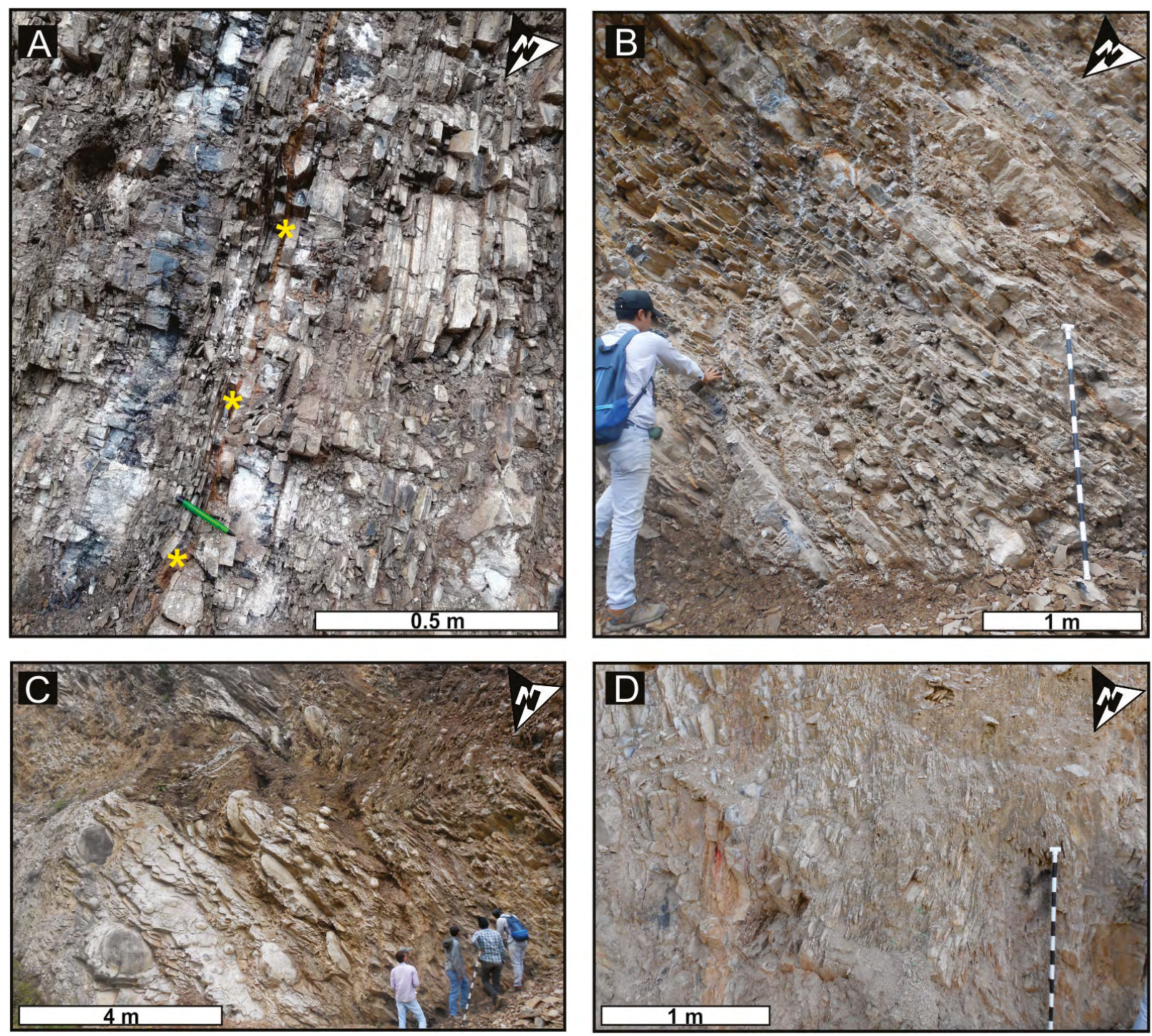

Figura 3. Sección de la Formación La Luna en "La Cantera" (Matanza, Santander). A. Niveles de chert en el Segmento I. Se indica un posible nivel de bentonitas (*). B. Mudstones fosfáticos con laminación planoparalela en la parte media del segmento II. C. Concreciones sobre la base del segmento III. D. Lodolitas calcáreas de geometría tabular (Segmento IV). Cada intervalo del bastón es igual a $10 \mathrm{~cm}$.

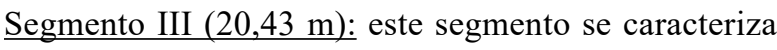
por una sucesión de estratos medios tabulares de lodolitas calcáreas laminadas inter-estratificadas con mudstones y wackestones color gris oscuro (N3). Algunos estratos presentan geometría lenticular. El desarrollo de concreciones esféricas de hasta 30 centímetros de diámetro es común en las lodolitas calcáreas. Las capas de mudstones ubicadas en la base del segmento presentan fragmentos de vertebrados (vértebras y escamas de peces) y mayor contenido de materia orgánica respecto a aquellas que se encuentran hacia el tope. 
En la parte media del segmento, las capas de wackestones contienen conchillas de foraminíferos (identificables con más detalle en sección delgada; Figura 4) y fosfatos color gris claro (N7). También se tienen capas de chert muy delgadas $(<10 \mathrm{~cm})$ con geometría irregular. En la parte superior del segmento las concreciones aumentan en tamaño y son más elongadas en el eje paralelo a la estratificación. Algunas de estas concreciones presentaron restos de amonitas (Figura 3C).

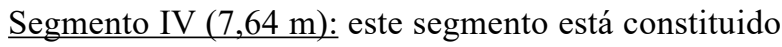
por capas delgadas y medias de wackestones y mudstones color gris oscuro a negro grisáceo ( $\mathrm{N} 2$ - N3) inter-estratificados con estratos de lodolitas calcáreas silicificadas de geometría tabular (Figura 3D). Las capas de wackestones presentan concreciones de 10 a $12 \mathrm{~cm}$ de diámetro, y en algunos casos presentan impregnaciones de hidrocarburos (muestra MAT-17). En las capas de mudstones se reconocieron conchas de bivalvos de tonalidad gris muy claro (N8), con un moderado grado de preservación, impresiones de conchas menores a un $1 \mathrm{~cm}$ y escamas de peces en menor cantidad. En algunas de las concreciones se encontraron restos de amonitas y conchillas de foraminíferos (Figura 4).

Las cinco muestras analizadas para petrografía, y distribuidas a lo largo de la sección, son definidas como biomicritas de foraminíferos (20-40\% de la composición total) con un alto grado de empaquetamiento, lo cual no permitió una clasificación taxonómica resolutiva (Figura 4).
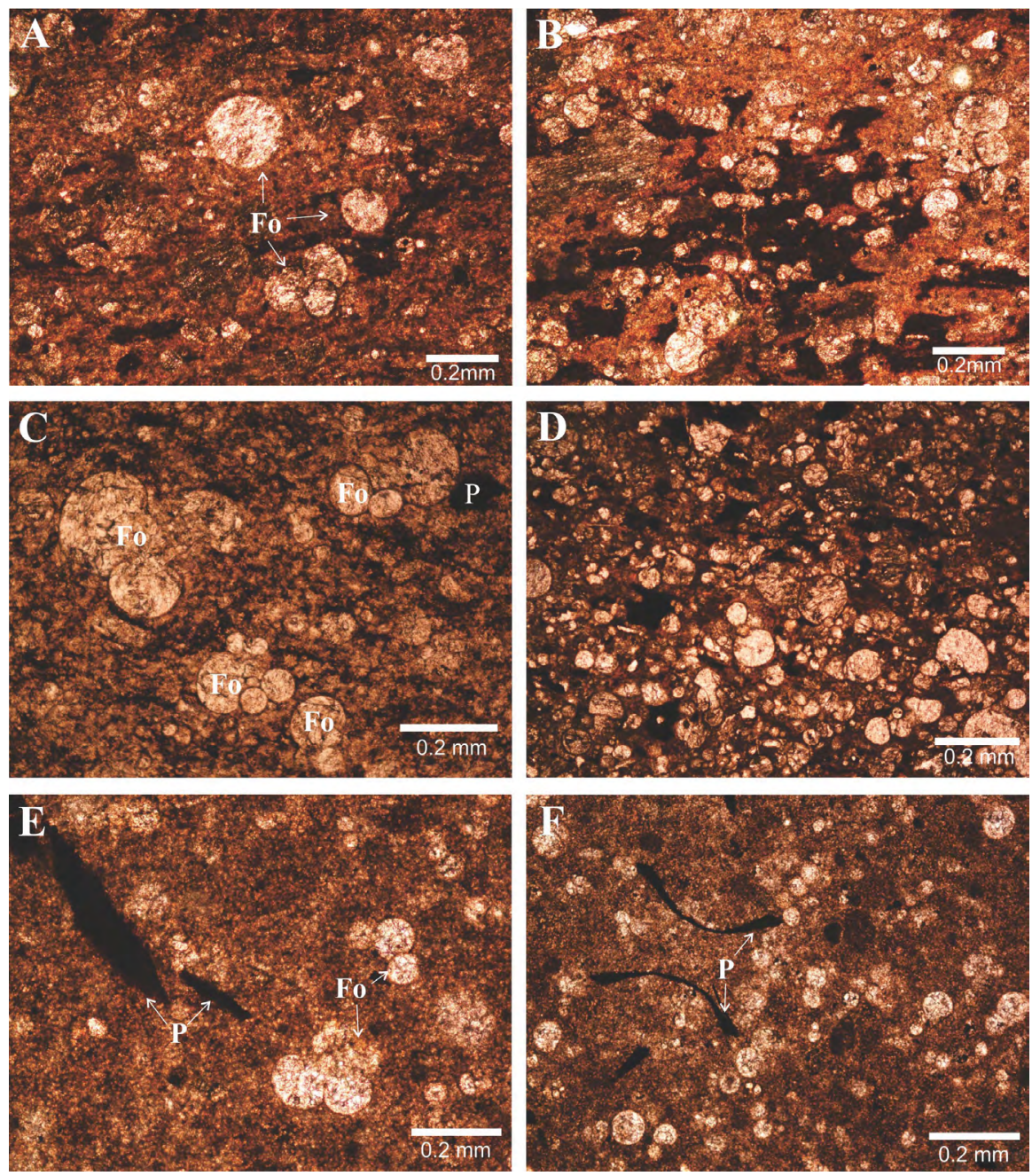

Figura 4. Petrografía de la Formación La Luna en la sección de Matanza. Fotografías en nicoles paralelos. Fo: Foraminíferos; P:

Fosfatos. A-B. Biomicrita de foraminíferos uniseriales, biseriales y trocoespiralados, con presencia de restos fosfáticos (muestra MAT-1). C-D. Biomicrita de foraminíferos trocoespiralados y uniseriales (muestras MAT-16 y MAT-7). E-F. Biomicrita de foraminíferos con fragmentos esqueletales fosfáticos de peces (muestra MAT-17). 
Se identificaron conchillas de foraminíferos con enrollamiento uniserial, biserial, y trocoespiral, siendo las dos últimas las más comunes dentro de las formas planctónicas. Las conchillas bentónicas, cuya abundancia fue muy baja en comparación a las formas planctónicas, correspondieron principalmente a formas biseriales, elongadas. Adicionalmente, dentro de los aloquímicos se identificaron fosfatos $(2-12 \%)$, materia orgánica amorfa y restos de peces $(>1 \%)$. Estos elementos se encuentran orientados en láminas planoparalelas que yacen en una matriz micrítica (50$80 \%$ ) con muy poca porosidad $(>1 \%)$.

\section{Paleontología}

En la prospección paleontológica se recolectaron invertebrados que corresponden a amonitas, bivalvos y gasterópodos. Entre las amonitas, predominan las de morfología discoidal (oxicónicas), con tamaños que oscilan entre 5 y $15 \mathrm{~cm}$ de diámetro y se asocian a los géneros Paralenticeras y Eulophoceras. La preservación y falta de diagnósticos en la zona umbilical no permiten una clasificación a nivel de especie (Figura 5C-5F).

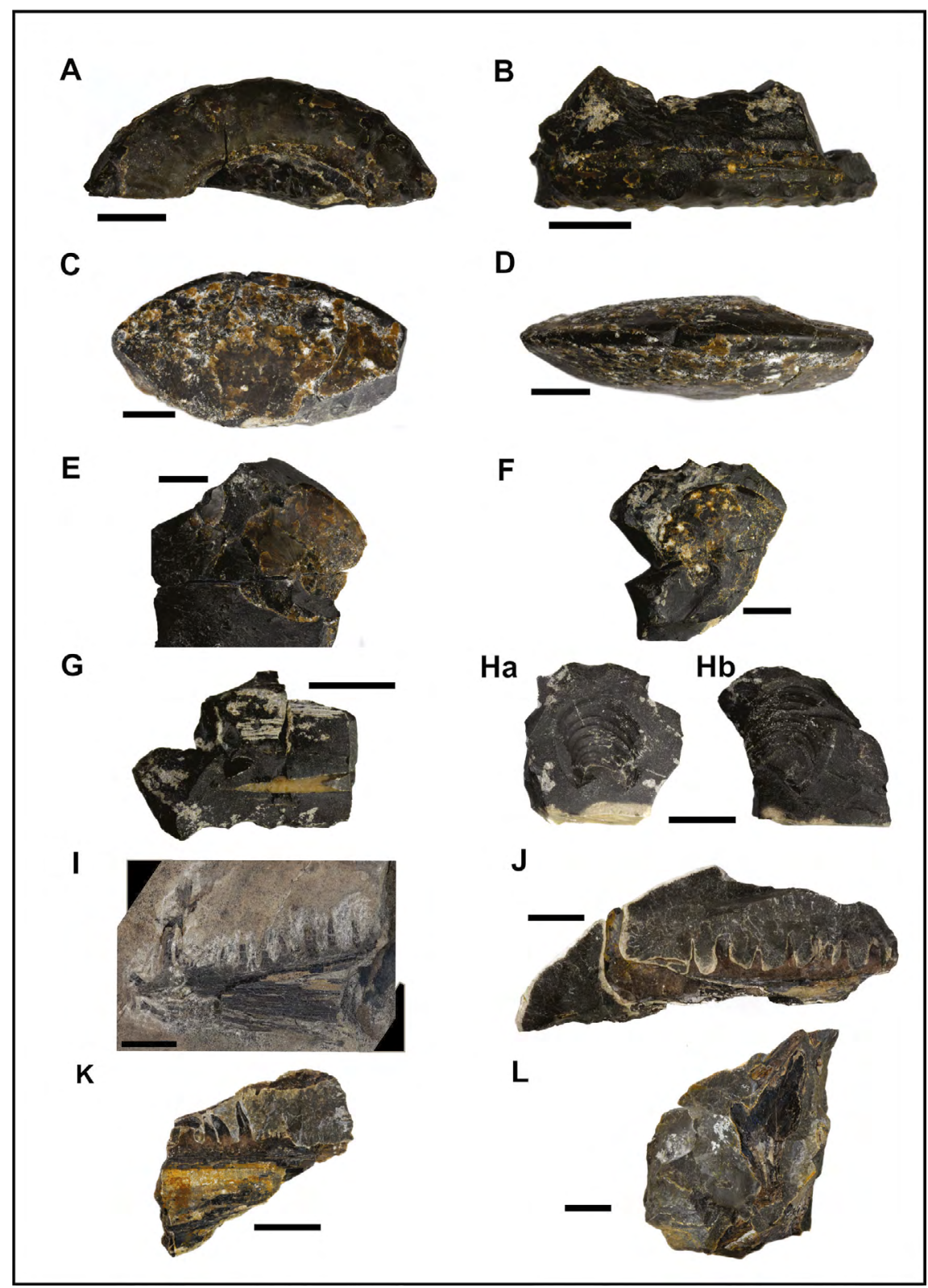

Figura 5. Fósiles representativos de la columna estratigráfica de la Formación La Luna en el sector de Matanza. A-B. Texanites? sp. C-D-E-F. Paralenticeras? sp.; Eulophoceras? sp. G. Baculites sp. Ha-b. Dydimotis? sp; Mytiloides? sp.; I-J-K. Dientes de peces (Enchodontoidei). L. Restos de aletas. Escala $2 \mathrm{~cm}$. 
También se colectaron amonitas de morfología serpenticónica que pertenecerían al género Texanites. El ejemplar corresponde a una forma incompleta, donde se logró identificar el desarrollo de la carena en la parte dorsal, costillas rectas con cuatro tubérculos igualmente espaciados que inician en la pared umbilical y terminan en la zona dorsolateral (Figura 5A-5B). Vestigios incompletos de líneas de sutura en la parte umbilical están presentes.

En menor proporción se encontraron amonitas baculicónicas de aproximadamente 3 a $4 \mathrm{~cm}$ de longitud y que podrían corresponder a Baculites inornatus?. Por último, se hallaron los inocerámidos Dydimotis? y Mytiloides? (Figura 5Ha-5Hb), al igual que gasterópodos como Turritella, con una longitud de 8-10 $\mathrm{mm}$.

Los restos de vertebrados corresponden a partes milimétricas desarticuladas de peces, principalmente escamas, vertebras, espinas y dientes (Figura 5I-5L). Adicionalmente, se han encontrado restos incompletos de dos peces, tanto en afloramiento, como en material rodado. Es posible distinguir una vértebra de morfología cónica y partes de su mandíbula con buena a moderada preservación de los dientes. Asimismo, se identificaron aletas, probablemente de la parte lateral. El segundo pez encontrado en la parte superior del Segmento IV, corresponde al fragmento de una mandíbula, con una preservación moderada a buena (Figura 5I-5J). La morfología de los aparatos bucales es semejante a las descritas en los enchodontoideos (Silva y Gallo, 2011). Los análisis micropaleontológicos de palinología y foraminíferos, presentaron resultados contrastantes en las muestras analizadas, que son las mismas estudiadas para petrografía. En general, la asociación de foraminíferos correspondió a conchillas planctónicas (completas y fragmentos de cámaras) de las familias Heterohelicidae y Hedbergellidae, en las que debido al alto grado de recristalización no fue posible llegar a nivel de género y especie. Otros microfósiles identificados correspondieron a restos de peces (muestra MAT-1). En las muestras de palinología resultó imposible el reconocimiento de elementos distintivos, dado el alto contenido de materia orgánica amorfa (Rueda, M., comunicación verbal).

\section{Geoquímica}

Los valores obtenidos de COT se aprecian en la Tabla 2 , los cuales varían en un rango entre $0,288 \%$ y $1,607 \%$, presentando un contenido promedio de $0,796 \%$.

Tabla 2. Valores de Carbono Orgánico Total (COT) en las muestras de la sección de Matanza. Potencial de hidrocarburos estimado según los rangos propuestos en Alexander et al. (2011).

\begin{tabular}{cccc}
\hline Muestra & Litología & COT $\mathbf{( \% P e s o})$ & Potencial \\
\hline MAT-2 & Mudstone & 1,200 & Medio \\
MAT-3 & Mudstone & 1,513 & Medio \\
MAT-4 & Mudstone & 0,406 & Muy pobre \\
MAT-6 & Lodolita calcárea & 0,750 & Pobre \\
MAT-10 & Lodolita calcárea & 0,470 & Muy pobre \\
MAT-13 & Mudstone & 0,446 & Muy pobre \\
MAT-14 & Chert & 0,288 & Muy pobre \\
MAT-16 & Mudstone & 0,733 & Pobre \\
MAT-17 & Wackestone & 1,607 & Medio \\
MAT-19 & Chert & 0,547 & Pobre \\
\hline
\end{tabular}

A partir de los resultados obtenidos y teniendo en cuenta los rangos de clasificación asignados para relacionar el COT con el potencial de hidrocarburos (Alexander et al., 2011), la riqueza de materia orgánica en esta sección de la Formación La Luna varía entre capacidad de generación pobre a media. La muestra con los valores de COT más altos, correspondió a una concreción calcárea con restos de bitumen (MAT-17).
A lo largo de la columna estratigráfica el COT es variable. Los valores más altos se registraron en las muestras del Segmento IV, mientras que las litologías de chert presentes en el Segmento III presentaron los valores de COT más bajos (Figura 2). 


\section{Discusión}

\section{Ambiente deposicional y modelo de edad}

A partir de las características texturales y composicionales del material sedimentario se propone que el ambiente de depositación de los sedimentos de la Formación La Luna, fue de plataforma externa, con condiciones de baja energía y un fondo de morfología llana. La presencia moderada a nula de restos fósiles epifaunales (bivalvos y foraminíferos bentónicos), y la ausencia de bioperturbación en las rocas sugieren condiciones de baja oxigenación en el fondo. Sin embargo, efectos diagenéticos y tectonismo también podrían explicar la ausencia de bioturbación en los estratos. El tipo de foraminíferos bentónicos encontrados (infaunales alargados), si bien escasos, junto con la presencia de los niveles fosfáticos del segmento II, apoyarían la presencia de fenómenos de surgencia. Las formas infaunales alargadas (bulimínidos), presentan una mayor tolerancia a entornos eutróficos, con baja oxigenación de fondo, y han sido reportados en distintas secciones del Cretácico superior en Colombia (Petters, 1955; Patarroyo et al., 2017). En general, las interpretaciones ambientales propuestas para la sección de Matanza, concuerdan con las propuestas por autores como Ward et al. (1973), Rangel et al. (2000), Sarmiento et al. (2015), TerrazaMelo (2019), entre otros, para el VMM, y las indicadas en estudios regionales en el sector de Maracaibo ( $L a$ Luna Sea; Martinez y Hernandez, 1992; Erlich et al., 1999, 2000; Sarmiento, 2018).

Por otro lado, el tipo de fauna encontrada en la sección de Matanza permite una definición cronoestratigráfica apropiada. El género Paralenticeras es diagnóstico del Coniaciano, aunque existen algunas especies que abarcan el Santoniano (Collignon, 1983; Kennedy et al., 1995). A su vez, tanto en Colombia como en Venezuela el género Eulophoceras se encuentra asociado a depósitos del Coniaciano (Renz, 1982; Villamil, 1998; Patarroyo y Dueñas, 2006) y las primeras especies del género Texanites se han utilizado para definir la base del Santoniano (Kennedy, 1984). Si se validara la presencia de Baculites inornatus entre el material encontrado, se corrobora una edad de Coniaciano (sensu Villamil, 1998) para la sección estudiada. En contraste, los bivalvos inocerámidos, que están representados por los géneros Dydimotis y Mytiloides, indican una edad de Santoniano (Villamil, 1998). Teniendo en cuenta los invertebrados identificados se sugiere una edad general de Coniaciano-Santoniano para la sección de Matanza.
Este rango de edad concuerda con lo observado en las asociaciones de foraminíferos encontradas. Si bien su preservación no es la ideal, la ausencia de formas características del Campaniano (Siphogenerinoides spp., Bolivina explicata; Petters, 1955; Patarroyo et al., 2017; Terraza-Melo, 2019) sugiere una edad no más joven que el Santoniano. Para el caso de los vertebrados encontrados en la sección, solo una apropiada clasificación taxonómica permitirá evaluar el potencial bioestratigráfico de los restos de peces. Aunque el grupo de los enchodontoideos presenta un rango bioestratigráfico bastante amplio (BarremianoEoceno temprano), fue durante el Cretácico Tardío que alcanzaron una amplia distribución geográfica, con reportes constantes a partir del Cenomaniano en México, Estados Unidos y Brasil (Silva y Gallo, 2011, 2016).

\section{Implicaciones estratigráficas}

Teniendo en cuenta las observaciones realizadas, el ambiente deposicional y el modelo de edad propuesto, la sección de Matanza presenta similitudes con el segmento superior de la Formación La Luna definido en el VMM y en la región de Maracaibo (Morales et al., 1958; Ward et al., 1973; Erlich et al., 1999, 2000; Rangel et al., 2000; Sarmiento et al., 2015; CasadiegoQuintero y Ríos-Reyes, 2016). Para el caso específico del VMM, las características litológicas y el tipo de fauna encontrada concuerdan con las descritas en la re-definición formal de la Formación Galembo por parte de Terraza-Melo (2019). En general, según ese autor dicha unidad se caracteriza por intercalaciones de mudstones, niveles fosfáticos, limolitas y capas delgadas de chert. Teniendo en cuenta su relativa cercanía geográfica y la descripción de secciones de referencia en el Valle Medio del Magdalena como las quebradas La Sorda y Aguablanca (Sarmiento et al., 2015; Casadiego-Quintero y Ríos-Reyes, 2016), se propone que el afloramiento estudiado en Matanza correspondería a la parte media a superior de la Formación Galembo (sensu Terraza-Melo, 2019). Como se refirió antes, este autor revalida las observaciones estratigráficas de Wheeler (1929), y muestra la inconveniencia del término "Formación La Luna" para el VMM, dada la heterogeneidad litológica que se reporta para dicha unidad en Venezuela.

Pero, ¿dicho argumento sería válido para secciones de la "Formación La Luna" que no hacen parte del VMM?, y ¿a partir de qué región se puede hablar de la Formación La Luna sensu stricto? 
Por ejemplo, la sección de Matanza también presenta similitudes en sus características litológicas y cronoestratigráficas con las del Miembro Timbetes de la Formación La Luna (Andes de Mérida), si solo se considerara la litoestratigrafía de la región de Maracaibo (Erlich et al., 1999). Esta ambigüedad en lo que se puede definir como "Formación La Luna", queda manifiesta en la forma como Ward et al. (1973) compila la información litoestratigráfica del Cretácico para los cuadrángulos H12 y H13 (ver descripciones de la sección de La Sorda y Quebrada El Pórtico; Lourdes, Norte de Santander).

Una forma de reforzar la necesidad de una estratigrafía clara en la sucesión sedimentaria cretácica del
Macizo de Santander es contextualizando los datos de geoquímica en la sección de Matanza. La Figura 6 muestra la distribución de los valores de Carbono Orgánico Total (COT) de occidente a oriente de la Formación Galembo sensu Terraza-Melo (2019) en diversas localidades: $\mathrm{km} 7$ vía Landázuri-Cimitarra, Quebrada El Salto, Quebrada Aguablanca, Quebrada La Sorda, Quebrada La Azufrada (Rangel et al., 2000; Carvajal, 2004; Martínez y Mendoza, 2012; TorresParada, 2008; Casadiego-Quintero y Ríos-Reyes, 2016). Asimismo, se muestran datos compilatorios del nororiente de la cuenca del Catatumbo (Pedraza y Ramírez, 2011).

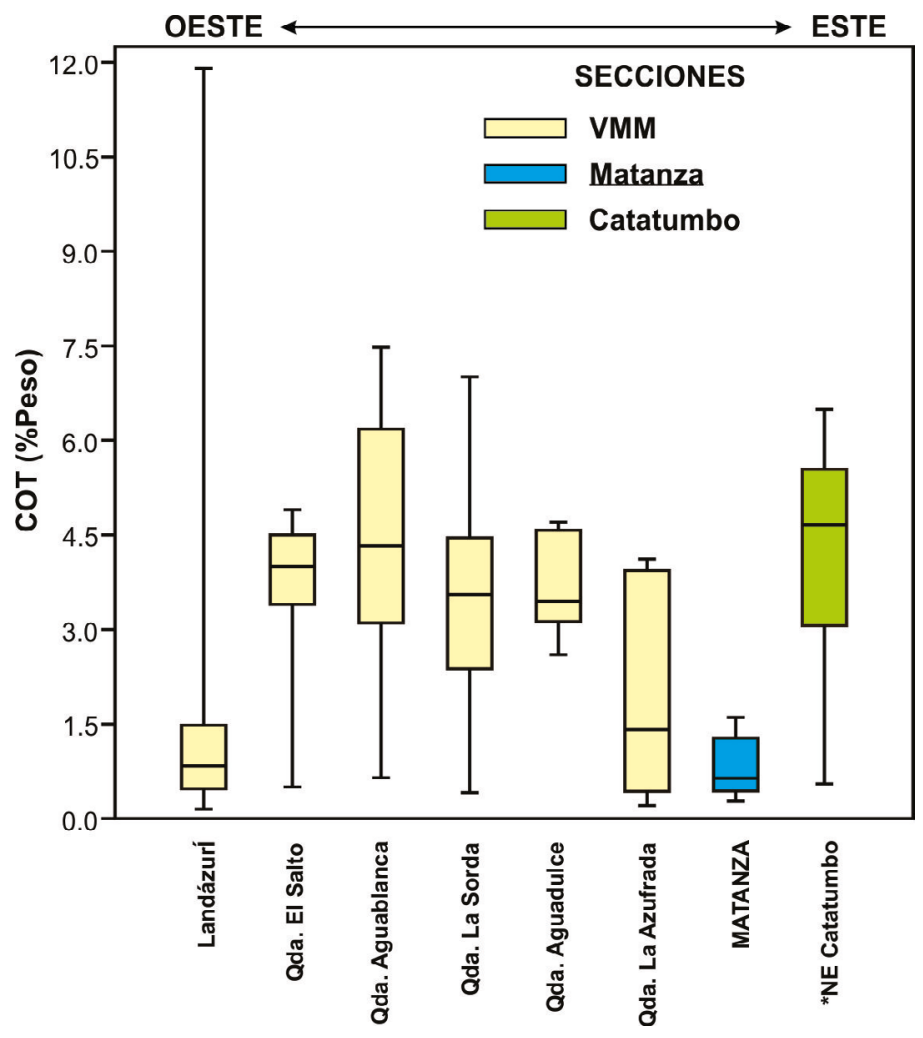

Figura 6. Valores de geoquímica de Matanza comparados con los reportados por otros autores para el Valle Medio del Magdalena (Rangel et al., 2000; Carvajal, 2004; Martínez y Mendoza, 2012; Torres-Parada, 2008; Casadiego-Quintero y Ríos-Reyes, 2016). *Se incluyen los valores regionales para la cuenca del Catatumbo (Pedraza y Ramírez, 2011).

En general, las estimaciones de COT en Matanza tienen valores dentro del rango descrito para la Formación Galembo y para la Formación La Luna del Catatumbo. Sin embargo, dichos valores son bajos, en comparación a aquellos presentes en los estudios del VMM, o los valores registrados para Venezuela $(2,5-10,8 \%$; Talukdar y Marcano, 1994). Una menor cantidad de datos en la sección de Matanza, al igual que una mayor madurez termal de la sucesión sedimentaria, producto del fuerte tectonismo en esta parte del macizo, pueden explicar los bajos valores de COT.

Teniendo en cuenta las observaciones previas, la mejor forma de aclarar el carácter estratigráfico de la sucesión de Matanza, es a través de un reconocimiento geológico detallado de este flanco del Macizo de Santander. El área de estudio presenta una complejidad estructural alta, debido a la influencia del sistema de fallas del Río 
Suratá, y por ejemplo en este trabajo no fue posible definir la naturaleza de los contactos estratigráficos con las unidades subyacentes y suprayacentes (formaciones Simití y Umir, de acuerdo a la cartografía de Ward et al., 1977). Teniendo en cuenta estos aspectos, es posible que el segmento estudiado esté incompleto, considerando que Terraza-Melo (2019) y otros autores reportan un espesor aproximado de 200 m para la Formación Galembo. Sin embargo, para los Andes de Mérida y la Serranía del Perijá, se registran variaciones significativas en el espesor de la Formación La Luna (100-300 m), teniendo por ejemplo un espesor promedio de $50 \mathrm{~m}$ en la cuenca del Catatumbo (Ward et al., 1973; Pedraza y Ramírez, 2011). Dichas variaciones han sido explicadas tanto por procesos erosivos o secciones condensadas hacia el tope de la formación (De Romero et al., 2003; Patarroyo et al., 2017). Por ende, solo la caracterización estratigráfica rigurosa de secciones con el registro sedimentario del Cretáceo completo, como el que se reporta sobre la vía que conduce de Suratá a California, ayudarán a determinar si el espesor estudiado en Matanza está completo. A su vez, el reconocimiento de unidades estratigráficas con un espesor discreto y extensión regional limitada como la Formación Calizas del Salto (Cenomaniano, VMM; Terraza-Melo, 2019) o el Miembro Tres Esquinas (Santoniano-Campaniano, Maracaibo; Erlich et al., 2000), ayudarían a determinar la afinidad litoestratigráfica de la sección de Matanza.

Por último, un aspecto con importancia estratigráfica, es la presencia de las capas de bentonita en la sección de Matanza, ya que también están presentes en los afloramientos de la Formación La Luna sobre la vía Suratá-California. A nivel regional, la presencia de bentonitas ha sido ampliamente reportada en las sucesiones del Cretácico superior en Colombia y Venezuela. Tal es el caso de Villamil (1998), el cual reporta múltiples eventos de formación de bentonitas, los cuales se distribuyeron desde el Cenomaniano hasta el Santoniano. Así mismo, Terraza-Melo (2012) reconoce niveles de cenizas volcánicas para el Albiano superior y Coniaciano medio en la Formación Areniscas de Chiquinquirá. En el caso puntual del VMM, niveles de bentonitas han sido reportados entre el TuronianoSantoniano en el pozo La Luna-1 (Ballesteros et al., 2013), al igual que en secciones de las formaciones Galembo y La Renta (Secciones Aguablanca y Montebello; Terraza-Melo, 2019). Finalmente, en la región de Maracaibo, los niveles de ceniza volcánica han sido reportados en estratos de edad CenomanianoTuroniano (Davis et al., 1999).
Al igual que la información preliminar de geoquímica que se discutió, la presencia o no de niveles de bentonitas en el flanco oeste del Macizo de Santander, no permite aclarar la afinidad estratigráfica de la sección de Matanza. Sin embargo, análisis geoquímicos y geocronológicos detallados de los niveles de bentonitas sí pueden proveer información de interés para el entendimiento de la evolución geológica de esta parte de la cordillera.

\section{Conclusiones}

El estudio geológico de una sección del Cretácico superior en el sector de Matanza (flanco oeste del Macizo de Santander), permitió las siguientes conclusiones:

La sección, que comprende una alternancia de mudstones y wackestones inter-estratificados, niveles de chert, fosforitas y bentonitas, fue depositada en un ambiente de plataforma externa. El tipo de microfósiles hallados (foraminíferos planctónicos biseriales, bentónicos infaunales), así como la ausencia de bioturbación de los sedimentitas, sugieren una baja oxigenación del fondo.

Respecto a la edad de la sucesión sedimentaria, las asociaciones paleontológicas indican un rango entre el Coniaciano y el Santoniano. Esto, teniendo en cuenta la presencia de los géneros Paralenticeras y Eulophoceras, asociados al Coniaciano, y el género Texanites, el cual es indicador de inicios del Santoniano. El potencial bioestratigráfico de los restos de vertebrados (Enchodontoidei) está pendiente de ser evaluado.

Teniendo en cuenta las características litológicas y geoquímicas descritas, al igual que el tipo de fauna hallada, se propone que la sección de la "Formación La Luna" en Matanza presenta similitudes con la parte superior de la Formación Galembo, siguiendo las observaciones estratigráficas más recientes para el Valle Medio del Magdalena.

Sin embargo, se recomienda la ampliación de los estudios estratigráficos en otras secciones reportadas de la Formación La Luna, y demás unidades del Cretácico para el flanco del oeste del Macizo de Santander. Esto, para poder determinar apropiadamente las relaciones litoestratigráficas que presenta la sucesión sedimentaria de esta zona, respecto a las cuencas del Valle Medio del Magdalena y del Catatumbo. 


\section{Agradecimientos}

Los autores de este trabajo agradecen al profesor Mario García y al Laboratorio de Geoquímica del Petróleo de la Universidad Industrial de Santander por la preparación de las muestras, al igual que al Laboratorio de Petrografía por el espacio. El material analizado para palinología fue gentilmente preparado y analizado por Milton Rueda (Paleoflora Limitada). Igualmente, el material paleontológico fue fotografiado por Gustavo Torres (Stratos Consultoría Geológica). Dos revisores aportaron comentarios y sugerencias para mejorar el manuscrito original.

\section{Referencias}

Alarcón, C.M.; Rodríguez, J.G. (2019). Estratigrafía, facies y paleoambiente de sedimentación de la Formación Jordán: un registro fluvio-lacustre con incidencia de actividad volcánica. Tesis de pregrado, Universidad Industrial de Santander, Bucaramanga, Colombia.

Alexander, T.; Baihly, J.; Boyer, C.; Clark, B.; Waters, G.; Jochen, V.; Le Calvez, J.; Lewis, R.; Miller, C.K.; Thaeler, J.; Toelle, B.E. (2011). Revolución del gas de lutitas. Oilfield Review, 23(3), 40-56.

Ballesteros, C.I.; Galvis, H.A.; Higuera, I.C.; Marfisi, N.K.; de la Parra, F.; Céspedes, S.; Cantisano, M.T.; Forero, S.P.; Barrera, P.A.; Rodríguez, C.E.; Zamora, W.H.; Restrepo, D.P.; Cerón, J.; Castillo, R.D. (2013). Anotaciones acerca de la estratigrafía del intervalo CenomanianoCampaniano atravesado por el pozo La Luna1 , cuenca del Valle Medio del Magdalena. XVI Congreso Colombiano de Geología, Bogotá, Colombia.

Bayona, G. (2018). El inicio de la emergencia en los Andes del norte: una perspectiva a partir del registro tectónico-sedimentológico del Coniaciano al Paleoceno. Revista de la Academia Colombiana de Ciencias Exactas, Físicas $y$ Naturales, 42(165), 364-378. https://doi. org/10.18257/raccefyn.632

Bayona, G.; Baquero, M.; Ramírez, C.; Tabares, M.; Salazar, A.M.; Nova, G.; Duarte, E.; Pardo, A.; Plata, A.; Jaramillo, C.; Rodríguez, G.; Caballero, V.; Cardona, A.; Montes, C.; Gómez-Marulanda, S.; Cárdenas-Rozo, A.L. (2020). Unravelling the widening of the earliest Andean northern orogen: Maastrichtian to early Eocene intrabasinal deformation in the northern Eastern Cordillera of Colombia. Basin Research. https:// doi.org/10.1111/bre.12496

Campbell, C. (1967). Lamina, laminaset, bed and bedset. Sedimentology, 8(1), 7-26. https://doi. org/10.1111/j.1365-3091.1967.tb01301.x

Caron, M. (1985). Cretaceous planktic foraminifera. In: H.M. Bolli; J. Saunders; K. Perch-Nielsen (eds). Plankton Stratigraphy (pp. 17-86). Cambridge University Press.

Carvajal, H. (2004). Nuevas consideraciones acerca del estado de madurez termal de la Formación La Luna en el Valle Medio del Magdalena, Colombia. Tesis. Universidad Industrial de Santander, Bucaramanga, Colombia.

Casadiego-Quintero, E.; Ríos-Reyes, C.A. (2016). Lithofacies analysis and depositional environment of the Galembo Member of La Luna Formation. CT\&F - Ciencia, Tecnología y Futuro, 6(4), 3756.

Choquette, P.; Pray, L. (1970). Geologic nomenclature and classification of porosity sedimentary carbonates. AAPG Bulletin, 54(2), 207-250. https://doi.org/10.1306/5D25C98B-16C1-11D7$8645000102 \mathrm{C} 1865 \mathrm{D}$

Collignon, M. (1983). Les faunesd'Ammonites du Santonien. En: M. Billotte, M. Collignon (eds). Biostratigraphie et Paléontologie des ammonites $\mathrm{du}$ Sénonien inférieur de Rennes-les-BainsSougraigne (Aude) [Zone sous-Pyrénéenne orientale]. Documents du Laboratoire de Géologie de Lyon, 6, 184-223.

Cooper, M.A.; Addison, F.T.; Alvarez, R.; Coral, M.; Graham, R.H.; Hayward, A.B.; Howe, S.; Martinez, J.; Naar, J.; Peñas, R.; Pulham, A.J.; Taborda, A. (1995). Basin development and tectonic history of the Llanos Basin, Eastern Cordillera, and Middle Magdalena Valley, Colombia. AAPG Bulletin, 79(10), 1421-1443. https://doi.org/10.1306/7834D9F4-1721-11D7$8645000102 \mathrm{C} 1865 \mathrm{D}$

Cordani, U.G.; Cardona, A.; Jiménez, D.M.; Liu, D.; Nutman, A.P. (2005). Geochronology of Proterozoic basement inliers in the Colombian 
Andes: tectonic history of remnants of a fragmented Grenville belt. Geological Society, London, Special Publications, 246, 329-346. https://doi.org/10.1144/GSL.SP.2005.246.01.13

Davis, C.; Pratt, L.; Sliter, W.; Mompart, L.; Murat, B. (1999). Factors influencing organic carbon and trace metal accumulation in the Upper Cretaceous La Luna Formation of the western Maracaibo Basin, Venezuela. In: E. Barrera; C.C. Johnson (eds). Evolution of the Cretaceous Ocean-climate System (pp. 203-230). Geological Society of America, Vol. 332 https://doi.org/10.1130/08137-2332-9.203

De Romero, L.; Truskowski, I.; Bralower, T.; Bergen, J.A.; Odreman, O.; Zachos, J.C.; Galea-Alvarez, F.A. (2003). An integrated calcareous microfossil biostratigraphic and carbon-isotope stratigraphic framework for the La Luna Formation, western Venezuela. Palaios, 18(4/5), 349-366.

Dörr, W.; Grösser, J.R.; Rodriguez, G.I.; Kramm, U. (1995). Zircon U-Pb age of the Paramo Rico tonalite-granodiorite, Santander Massif (Cordillera Oriental, Colombia) and its geotectonic significance. Journal of South American Earth Sciences, 8(2), 187-194. https:// doi.org/10.1016/0895-9811(95)00004-Y

Dunham, R.I. (1962). Classification of carbonate rocks according to depositional textures. In: W.E. Ham (ed.). Classification of carbonate rocks (pp. 108-121). Memoir I of American Association of Petroleum Geologists.

Erlich, R.N.; Macsotay, O.; Nederbragt, A.J.; Lorente, M.A. (1999). Palaeoecology, paleogeography and depositional environments of Upper Cretaceous rocks of western Venezuela. Palaeogeography, Palaeoclimatology, Palaeoecology, 153(14), 203-238. https://doi.org/10.1016/S00310182(99)00072-3

Erlich, R.N.; Macsotay, O.; Nederbragt, A.; Lorente, M.A. (2000). Birth and death of the Late Cretaceous "La Luna Sea", and origin of the Tres Esquinas phosphorites. Journal of South American Earth Sciences, 13(1-2), 21-45. https://doi.org/10.1016/S0895-9811(00)00016-X
Etayo-Serna, F. (1985). Metodología de trabajo del Proyecto Cretácico. En: F. Etayo-Serna; F. Laverde-Montaño (Eds.). Proyecto Cretácico (pp. I-1-I-13). INGEOMINAS, No. 16.

Etayo-Serna, F.; Rodríguez, G. (1985). Edad de la Formación Los Santos. En: F. Etayo-Serna; F. Laverde-Montaño (eds.). Proyecto Cretácico (pp. XXVI-1-XXVI-13). INGEOMINAS, No. 16.

Folk, R. (1962). Spectral subdivision of limestone types. In: W.E. Ham (ed.). Classification of carbonate rocks (pp. 62-84). Memoir I of American Association of Petroleum Geologists.

Garner, A.H. (1926). Suggested nomenclature and correlation of geological formations in Venezuela: Petroleum Development and Technology in 1925. Symposiums of the Petroleum Division.

Georgescu, M.D. (2005). On the systematics of rugoglobigerinids (Planktonic foraminifera, late Cretaceous). Studia Geologica Polonica, 124, 8797.

Goddard, E. (2009). Rock Color Chart Committee Geological Society of America. Rock-color chart. Grand Rapids: Geological Society of America.

Goldsmith, R.; Marvin, R.F.; Mehnert, H.H. (1971). Radiometric ages in the Santander Massif, Eastern Cordillera, Colombian Andes. U.S. Geological Survey Professional Paper 750-D: D44-D49.

Kammer, A.; Sánchez, J. (2006). Early Jurassic rift structures associated with the Soapaga and Boyacá faults of the Eastern Cordillera, Colombia: Sedimentological inferences and regional implications. Journal of South American Earth Sciences, 21(4), 412-422. https://doi. org/10.1016/j.jsames.2006.07.006

Kennedy, W.J. (1984). Ammonite faunas and the 'standard zones' of the Cenomanian to Maastrichtian Stages in their type areas, with some proposals for the definition of the stage boundaries by ammonites. Bulletin of the Geological Society of Denmark, 33(1-2), 147-163.

Kennedy, W.J.; Bilotte, M.; Melchior, P. (1995). Ammonite faunas, biostratigraphy and sequence 
stratigraphy of the Coniacian-Santonian of the Corbières (NE Pyrénées). Bulletin des Centres de Recherches Exploration-Production Elf Aquitane, 19(2), 377-499.

Machado, M.C.; Chemale Jr. F.; Kawashita, K.; Rey, O.; Moura, C.A.V. (2016). Isotope studies of carbonate rocks of La Luna Formation (Venezuela) to constrain the oceanic anoxic event 3 (OAE3). Journal of South American Earth Sciences, 72, 3848. https://doi.org/10.1016/j.jsames.2016.07.001

Mann, P.; Escalona, A.; Castillo, M.V. (2006). Regional geologic and tectonic setting of the Maracaibo supergiant basin, western Venezuela. AAPG Bulletin, 90(4), 445-477. https://doi. org/10.1306/10110505031

Mantilla-Figueroa, L.C.; Bissig, T.; Valencia, V.; Hart, C.J. (2013). The magmatic history of the VetasCalifornia mining district, Santander massif, Eastern Cordillera, Colombia. Journal of South American Earth Sciences, 45, 235-249. https:// doi.org/10.1016/j.jsames.2013.03.006

Mantilla-Figueroa, L.C.; García-Ramírez, C.A.; Valencia, V.A. (2016). Propuesta de escisión de la denominada 'Formación Silgará' (Macizo de Santander, Colombia), a partir de edades $\mathrm{U}-\mathrm{Pb}$ en circones detríticos. Boletín de Geología, 38(1), 33-50. https://doi.org/10.18273/revbol.v38n12016002

Martinez, J.I.; Hernandez, R. (1992). Evolution and drowning of the Late Cretaceous Venezuelan carbonate platform. Journal of South American Earth Sciences, 5(2), 197-210. https://doi. org/10.1016/0895-9811(92)90038-Z

Martínez, D.; Mendoza, C. (2012). Definición de facies generadoras para la Formación La Luna en la parte noroeste de la cuenca del Valle Medio del Magdalena. Tesis. Universidad Industrial de Santander, Bucaramanga, Colombia.

Mojica, J.; Kammer, A. (1995). Eventos jurásicos en Colombia. Geología Colombiana, 19, 165-172.

Mojica, J.; Kammer, A.; Ujueta, G. (1996). El Jurásico del sector noroccidental de Suramérica y guía de la excursión al Valle Superior del Magdalena (Nov 1-4/95), regiones de Payandé y Prado,
Departamento del Tolima, Colombia. Geología Colombiana, 21, 3-41.

Morales, L.G.; Podesta, D.J.; Hatfield, W.C.; Tanner, H.; Jones, S.H.; Barker, M.H.; O’Donoghue, D.J.; Moler, C.E.; Dubois, E.P.; Jacobs, C.; Goss, C.R. (1958). General geology and oil occurrences of the Middle Magdalena Valley, Colombia. In: L.G. Weeks (ed.). Habitat of Oil (pp. 641-695). AAPG.

Nederbragt, A. (1991). Late Cretaceous biostratigraphy and development of Heterohelicidae (planktic foraminifera). Micropaleontology, 37(4), 329372. https://doi.org/10.2307/1485910

Patarroyo, P.; Dueñas, H. (2006). Eulophoceras jacobi Hourcq y palinomorfos asociados del Coniaciano temprano, Formación Loma Gorda, Quebrada Bambucá (Aipe-Huila-Colombia, S.A.). Revista de la Academia Colombiana de Ciencias Exactas, Físicas y Naturales, 30(117), 503-510.

Patarroyo, P. (2011). Sucesión de amonitas del Cretácico Superior (Cenomaniano-Coniaciano) de la parte más alta de la Formación Hondita y de la Formación Loma Gorda en la Quebrada Bambucá, Aipe-Huila (Colombia, S. A.). Boletín de Geología, 33(1), 69-92.

Patarroyo, G.D.; Torres, G.A.; Rincón, D.A.; Cárdenas, C.P.; Márquez, R.E. (2017). Bioestratigrafía e inferencias paleoambientales de las asociaciones de foraminíferos en las formaciones cretácicas $\mathrm{La}$ Luna-Colón (Cuenca del Catatumbo, Colombia). Boletín de Geología, 39(3), 25-40. https://doi. org/10.18273/revbol.v39n3-2017002

Pedraza, P.; Ramírez, Y. (2011). Catatumbo Basin. In: F. Cediel; F. Colmenares (eds.). Petroleum Geology of Colombia. Volume 5. ANH-Fondo Editorial Universidad EAFIT.

Petters, V. (1955). Development of Upper Cretaceous foraminiferal faunas in Colombia. Journal of Paleontology, 29(2), 212-225.

Premoli-Silva, I.; Verga, D. (2011). Practical manual of Cretaceous planktonic foraminifera. New Editing by Marie Rose Petrizzo and Isabella Premoli Silva. International School on Planktonic foraminifera 3rd Course: Cretaceous Planktonic Foraminifera. Perugia. 
Rangel, A.; Parra, P.; Niño, C. (2000). The La Luna formation: chemostratigraphy and organic facies in the Middle Magdalena Basin. Organic Geochemistry, 31(12), 1267-1284. https://doi. org/10.1016/S0146-6380(00)00127-3

Renz, O. (1960). Geología de la parte sureste de la Península de La Guajira (República de Colombia). Congreso Geológico Venezolano, Venezuela.

Renz, O. (1982). Cretaceous Ammonites of Venezuela. Birk Hause Ed.

Restrepo-Pace, P.A.; Cediel, F. (2010). Northern South America basement tectonics and implications for paleocontinental reconstructions of the Americas. Journal of South American Earth Sciences, 29(4), 764-771. https://doi.org/10.1016/j. jsames.2010.06.002

Santana, J.O. (1986). Estratigrafía, facies y ambiente de depósito de la Formación Tambor en las localidades de Curití, Charta y Matanza, Santander. Tesis de pregrado. Universidad Nacional de Colombia, Bogotá, Colombia.

Sarmiento, L.F. (2011). Eastern Cordillera Basin. In: F. Cediel; F. Colmenares (eds.). Petroleum Geology of Colombia Volume 7. ANH-Fondo Editorial Universidad EAFIT.

Sarmiento, L.F. (2018). Cretaceous Stratigraphy and paleo-facies maps of northwestern South America. In: F. Cediel; R.P. Shaw (eds.). Geology and Tectonics of Northwestern South America. The Pacific Caribbean Andean Junction (pp. 673-747). Springer, Cham. https://doi.org/10.1007/978-3319-76132-9_10

Sarmiento, G.; Puentes, J.; Sierra, C. (2015). Evolución geológica y estratigrafía del sector norte del Valle Medio del Magdalena. Geología Norandina, 12, 51-82.

Silva, H.; Gallo, V. (2011). Taxonomic review and phylogenetic analysis of Enchodontoidei (Teleostei: Aulopiformes). Anais da Academia Brasileira de Ciências, 83(2), 483-511. https:// doi.org/10.1590/S0001-37652011000200010

Silva, H.; Gallo, V. (2016). Distributional patterns of enchodontoid fishes in the Late Cretaceous.
Cretaceous Research, 65, 223-231. https://doi. org/10.1016/j.cretres.2016.03.009

Sohn, I.G. (1961). Techniques for preparation and study of fossil ostracodes. In: R. Moore (ed.). Treatise on Invertebrate Paleontology (pp. Q63-Q70). Part Q, Arthropod 3. Geological Society of America \& University of Kansas Press.

Talukdar, S.; Marcano, F. (1994). Petroleum System of the Maracaibo Basin, Venezuela. In: L.B. Magoon; W.G. Dow (eds.). The petroleum system-from source to trap (pp. 463-481). AAPG Memoir, 60. https://doi.org/10.1306/M60585C29

Terraza-Melo, R. (2012). Estratigrafía y ambientes de depósito de la Arenisca de Chiquinquirá en los alrededores de la localidad tipo. Boletín de Geología, 34(2), 55-72.

Terraza-Melo, R. (2019). 'Formación La Luna': expresión espuria en la geología colombiana. En: F. Etayo-Serna (ed.). Estudios geológicos y paleontológicos sobre el Cretácico en la región del embalse del río Sogamoso, Valle Medio del Magdalena (pp. 305-362). vol. XXIII. Servicio Geológico Colombiano.

Torres-Parada, E. (2008). Interpretación sísmica, estructural y modelamiento geoquímico de la Formación La Luna, en el noreste de Colombia. Trabajo de grado. Universidad Industrial de Santander, Bucaramanga, Colombia.

Traverse, A. (2007). Paleopalynology. Second edition. Springer.

Van der Lelij, R. (2013). Reconstructing north-western Gondwana with implications for the evolution of the Iapetus and Rheic Oceans: a geochronological, thermochronological and geochemical study. PhD Thesis, University of Geneva, Switzerland. https:// doi.org/10.13097/archive-ouverte/unige:31653

Vargas, R.; Arias, A.; Jaramillo, L.; Téllez, N. (1981). Geología del cuadrángulo I-13, Málaga. Boletín Geológico, 24(3), 1-76.

Vásquez, M.; Altenberger, U.; Romer, R.L.; Sudo, M.; Moreno-Murillo, J.M. (2010). Magmatic evolution of the Andean Eastern Cordillera of Colombia during the Cretaceous: Influence of 
previous tectonic processes. Journal of South American Earth Sciences, 29(2), 171-186. https:// doi.org/10.1016/j.jsames.2009.02.003

Villamil, T. (1998). Chronology, relative sea level history and a new sequence stratigraphic model for Basinal Cretaceous Facies of Colombia. In: J.L. Pindell; C. Drake (eds.) Paleogeographic evolution and non-glacial eustacy, northern South America (pp. 161-216). SEPM Society for Sedimentary Geology, Vol. 58. https://doi. org/10.2110/pec.98.58.0161

Villamil, T.; Arango C. (1998). Integrated Stratigraphy of latest Cenomanian and Early Turonian Facies of Colombia. In: J. Pindell; C. Drake (eds.). Paleogeographic evolution and non-glacial eustacy, northern South America (pp. 129-159). SEPM Society for Sedimentary Geology, Vol. 58. https://doi.org/10.2110/pec.98.58.0129

Villamil, T.; Pindell, J. (1998). Mesozoic paleogeographic evolution of northern South America: foundations for sequence stratigraphic studies in passive margin strata deposited during non-glacial times. In: J. Pindell; Drake, C. (eds.). Paleogeographic evolution and non-glacial eustacy, northern South America (pp. 283-318). SEPM Society for Sedimentary Geology, Vol. 58. https://doi.org/10.2110/pec.98.58.0283
Villamil, T.; Arango, C.; Hay, W.W. (1999). Plate tectonic paleoceanographic hypothesis for Cretaceous source rocks and cherts of northern South America. In: E. Barrera; C.C. Johnson (eds.). Evolution of the Cretaceous OceanClimate System (pp. 191-202). Geological Society of America, vol. 332. https://doi.org/10.1130/08137-2332-9.191

Ward, D.E.; Goldsmith, R.; Cruz, J.; Restrepo, H. (1973). Geología de los cuadrángulos H-12 Bucaramanga y H-13 Pamplona, Departamento de Santander. Boletín Geológico, 21(1-3), 1-134.

Ward, D.E.; Goldsmith, R.; Jimeno, A.; Cruz, J.; Restrepo, H.; Gómez, E. (1977). Geologic Map of the Bucaramanga Quadrangle (H12), Colombia. US Geological Survey. https://doi.org/10.3133/ i1005

Wheeler, O.C. (1929). Report on the Palmira Series with notes on stratigraphy of the Umir, Lisama y La Paz Formations near the eastern part of De Mares Concession. Informe Geológico 37. Ecopetrol.

Fecha de recibido: 17 de abril de 2020

Fecha de aprobado: 03 de noviembre de 2020 